\title{
Ratiometric bioluminescent sensors towards in vivo imaging of bacterial signaling
}

Dippel, A. B*a,b, Anderson, W. A.*a,b, Park, J. H. ${ }^{c}$, Yildiz, F. H. ${ }^{c}$, and Hammond, M.C. ${ }^{a, b}$ *authors contributed equally

Author Affiliations:

a Department of Chemistry, University of California, Berkeley, Berkeley, California, USA.

b Department of Chemistry and Henry Eyring Center for Cell and Genome Sciences, University of Utah, Salt Lake City, UT, USA.

c Department of Microbiology and Environmental Toxicology, University of California, Santa Cruz, Santa Cruz, California, USA 


\section{ABSTRACT}

Second messenger signaling networks allow cells to sense and adapt to changing environmental conditions. In bacteria, the nearly ubiquitous second messenger molecule cyclic di-GMP coordinates diverse processes such as motility, biofilm formation, and virulence. In bacterial pathogens, these signaling networks allow the bacteria to survive changing environment conditions that are experienced during infection of a mammalian host. While studies have examined the effects of cyclic di-GMP levels on virulence in these pathogens, it has previously not been possible to visualize cyclic di-GMP levels in real time during the stages of host infection. Towards this goal, we generate the first ratiometric, chemiluminescent biosensor scaffold that selectively responds to c-di-GMP. By engineering the biosensor scaffold, a suite of Venus-YcgR-NLuc (VYN) biosensors is generated that provide extremely high sensitivity $\left(\mathrm{K}_{\mathrm{D}}<300 \mathrm{pM}\right)$ and large BRET signal changes (up to $109 \%$ ). As a proof-of-concept that VYN biosensors can image cyclic diGMP during host infection, we show that the VYN biosensors function in the context of a tissue phantom model, with only $\sim 10^{3}-10^{4}$ biosensor-expressing cells required for the measurement. Furthermore, the stable BRET signal suggests that the sensors could be used for long-term imaging of cyclic di-GMP dynamics during host infection. The VYN sensors developed here can serve as robust in vitro diagnostic tools for high throughput screening, as well as genetically encodable tools for monitoring the dynamics of c-di-GMP in live cells, and lay the groundwork for live cell imaging of c-di-GMP dynamics in bacteria during host infection, and other complex environments.

\section{INTRODUCTION}

The second messenger molecule cyclic di-GMP (c-di-GMP) is a key regulator of bacterial physiology and behavior, coordinating diverse processes such as motility, biofilm formation, and virulence. First discovered as a stimulator of cellulose synthesis, ${ }^{1} \mathrm{c}$-diGMP has since been found to be nearly ubiquitous in bacteria, with c-di-GMP signaling pathways often integrated with other global regulatory systems, such as phosphorylation networks and quorum sensing pathways. ${ }^{2,3}$ The intracellular levels of c-di-GMP are tightly regulated by diguanylate cyclase (DGC) and phosphodiesterase (PDE) enzymes that synthesize and degrade c-di-GMP, respectively. Many bacteria have an abundance of predicted DGC and PDE genes, suggesting unique c-di-GMP regulatory circuits are activated in response to different environmental cues. In many bacterial pathogens, including Pseudomonas aeruginosa, Clostridium difficile, Vibrio cholerae, and pathogenic strains of Escherichia coli, these complex c-di-GMP signaling networks allow the bacteria to adapt to and survive in the changing environmental conditions that are experienced during infection of a mammalian host. ${ }^{4}$ While multiple studies have examined the effects of c-di-GMP levels on virulence in these pathogens, there currently are no tools available that allow for the quantification of c-di-GMP in bacteria during stages of mammalian host infection. To interrogate these complex c-di-GMP signaling networks in bacteria over the course of the infection process, new analytical tools are needed for quantifying and imaging intracellular c-di-GMP levels within tissue over extended time frames.

Commonly used tools for analyzing intracellular c-di-GMP levels include phenotypic screens and mass spectrometry (MS) analysis of bacterial cell extracts. Phenotypic screens for motility and biofilm formation can serve as proxies for measuring intracellular 
c-di-GMP levels. 5,6 These assays can be high throughput and are useful in screening genetic knockouts, however they have low sensitivity and provide indirect measurement of c-di-GMP that can be complicated by pleiotropic effects. MS-based analysis of c-diGMP from bacterial cell extracts is highly sensitive and quantitative, however the multistep sample preparation and long analysis time required leads to reduced throughput. ${ }^{7-}$ 10 In addition, neither phenotypic assays nor mass spectrometry-based analysis can provide real-time, dynamic measurements of c-di-GMP in cells. To overcome these issues, our lab and others have developed several genetically encodable fluorescent biosensors that can report on single-cell dynamics of c-di-GMP using fluorescence microscopy or flow cytometry. ${ }^{11-13}$ These tools are sensitive, can provide real-time measurements of c-di-GMP dynamics, and are amenable to high throughput screening. Notable examples include protein-based FRET biosensors that have been used to image c-di-GMP dynamics during asymmetric cell division in Caulobacter crescentus ${ }^{11}$ and RNA-based fluorescent sensors that were used to visualize c-di-GMP changes in E. coli in direct response to an environmental signal, zinc. ${ }^{14}$

One drawback of fluorescent biosensors, however, is that due to a reliance on external illumination, these systems are incompatible with imaging in deep tissues of animals and in long-term experiments due to phototoxicity and/or photobleaching. In a preliminary effort to expand the capabilities of genetically encodable tools for quantifying c-di-GMP levels to overcome these issues, our lab developed the first chemiluminescent biosensors for c-di-GMP ${ }^{15}$ based on the yellow Nano-lantern (YNL) scaffold and a CSL-BRET mechanism. ${ }^{16}$ These YNL-YcgR biosensors provide nanomolar sensitivity for c-di-GMP with high selectivity, large signal changes, and a luminescent signal that is produced without external illumination. The sensors were used to develop a rapid, plate-reader based assay for measuring diguanylate cyclase activity in bacterial lysates. The intensitybased signal of these sensors is useful for in vitro activity assays with lysates or purified enzymes where biosensor and luminescent substrate levels can be controlled. However, in long-term imaging experiments and/or situations where luminescent substrate availability differs between samples, signal quantitation becomes complicated for intensity-based sensors. Accordingly, we encountered issues when applying the YNL sensors to live cell measurements of c-di-GMP in bacteria. ${ }^{15}$

Ratiometric BRET sensors using the engineered marine luciferase NanoLuc (NLuc) ${ }^{17}$ have recently been developed for imaging $\mathrm{Ca}^{2+},{ }^{18} \mathrm{Zn}^{2+},{ }^{19}$ and membrane voltage, ${ }^{20}$ however to our knowledge no sensors of this type have been applied to imaging in bacteria to date. In this work, we generate a suite of BRET biosensors that selectively respond to c-di-GMP and produce ratiometric signal changes. The tVYN-Tm $\Delta$ biosensor was applied in a plate reader-based assay to quantify c-di-GMP levels in $V$. cholerae extracts grown under a variety of conditions that mimic the infection cycle, with quantitation and sensitivity comparable to MS-based methods (LOD = $30 \mathrm{fmol}$ ). We also show that luminescent biosensors producing ratiometric-based signal enable long-term, quantitative, live cell imaging of c-di-GMP activity in E. coli using an IVIS small animal imaging system. 


\section{RESULTS AND DISCUSSION}

Design of BRET sensor for cyclic di-GMP. The starting BRET scaffold, V-NLuc, pairs the newly developed marine luciferase, NanoLuc (NLuc), with a truncation of the monomeric yellow fluorescent protein, Venus, as the donor and acceptor moieties, respectively, similar to the previously developed YeNL. ${ }^{21}$ NLuc produces a glow-type luminescence with an emission maximum at $460 \mathrm{~nm}$ and an overall luminescent output $\sim 100-150 x$ that of the commonly used Renilla or firefly luciferases. ${ }^{17}$ Compared to the intensity-based yellow Nano-lantern (YNL) sensors for c-di-GMP previously designed by our lab that use a mutated version of Renilla luciferase as the donor moiety, the substitution of NLuc should produce significantly higher signal intensity, improved thermodynamic stability, and increased signal stability over time. The emission of NLuc overlaps well with the excitation of Venus, producing an efficient energy transfer in VNLuc, as measured by the BRET ratio $(530 / 460 \mathrm{~nm})$ (Figure 1a).

To design a c-di-GMP sensor, a c-di-GMP-binding YcgR protein is inserted between Venus and NLuc (Figure 1b). YcgR-like proteins contain the c-di-GMP-binding PilZ domain at their $\mathrm{C}$-terminus. ${ }^{22-24}$ These proteins typically undergo large conformational changes upon c-di-GMP binding, ${ }^{24}$ which has been harnessed for the generation of genetically encoded sensors for c-di-GMP. ${ }^{11,12,15,25}$ Thus, binding of c-di-GMP to a VenusYcgR-NLLuc (VYN) sensor should produce a change in energy transfer efficiency between Venus and NLuc, changing the BRET ratio. The change could be from low to high BRET ratio upon binding, or vice versa, thereby producing positive or negative signal changes, respectively (Figure 1b).

For initial testing of the sensor design, full-length $\mathrm{YcgR}$ protein from Escherichia coli $(E c Y c g R)$ was inserted into the BRET scaffold to generate VYN-Ec. The sensor was purified from $E$. coli after co-expression with the c-di-GMP-specific phosphodiesterase (PDE) PdeH to ensure the sensor did not co-purify with endogenous c-di-GMP. The purified sensor showed a c-di-GMP dependent change from high to low BRET state (Figure 1c). Promisingly, the BRET ratio of VYN-Ec remained stable over time even as overall signal intensity decreased due to consumption of luminescent substrate (Figure S1a, b). VYN-Ec binds to two molecules of c-di-GMP with an apparent affinity (KD) of 50 $\mathrm{nM}$ and a BRET signal change of $-48 \%$ (Figure 1d). The sensor retains selectivity for cdi-GMP over structurally related cyclic dinucleotides (Figure S1c, d).

The affinity of the VYN-Ec sensor $\left(K_{D} \sim 50 \mathrm{nM}\right)$ is significantly higher compared to the equivalent $\mathrm{YNL}-E c \mathrm{YcgR}$ sensor $\left(\mathrm{K}_{\mathrm{D}} \sim 350 \mathrm{nM}\right)^{15}$ and previously reported affinity values for $E c Y c g R\left(K_{D} \sim 800 \mathrm{nM}\right) .{ }^{22}$ Since c-di-GMP binding to PilZ domains has been found to be largely entropically driven, ${ }^{24}$ this finding suggests that the VYN scaffold itself may be providing a degree of additional stability that results in a decreased entropic cost of binding C-di-GMP, leading to increased binding affinity. Encouraged by the VYN-Ec results, we sought to further improve the properties of the sensor via phylogenetic screening and semi-rational protein engineering. 


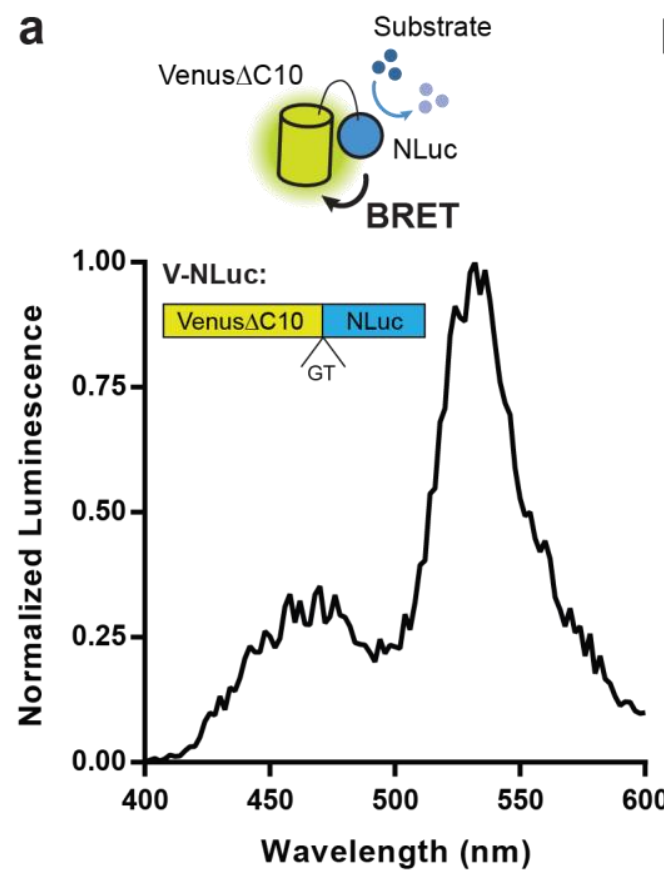

b

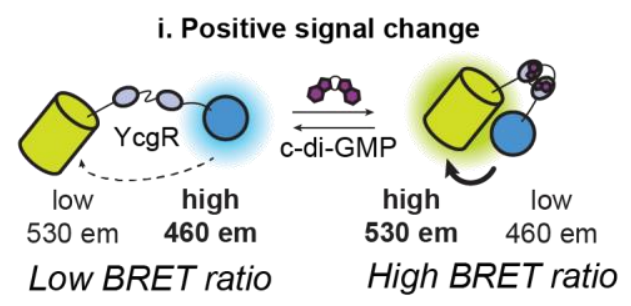

C

VYN-Ec:

d

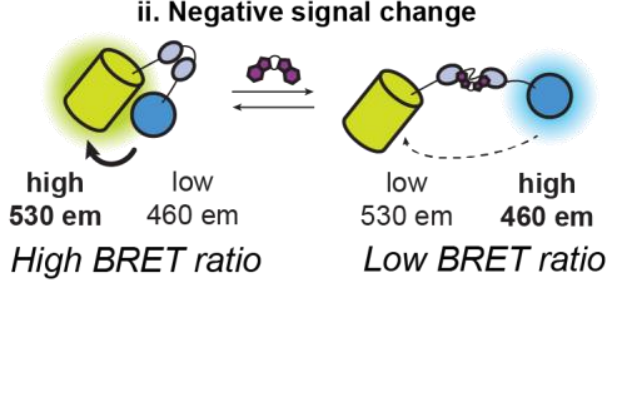

ii. Negative signal change

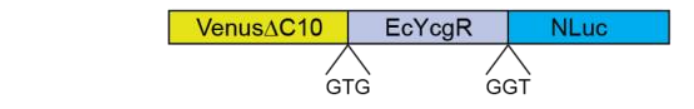
d $\quad K_{0}: 50 \pm 4 n M$
Hill slope: $-1.5 \pm 0.2$
$\triangle$ BRET ratio: -0.58
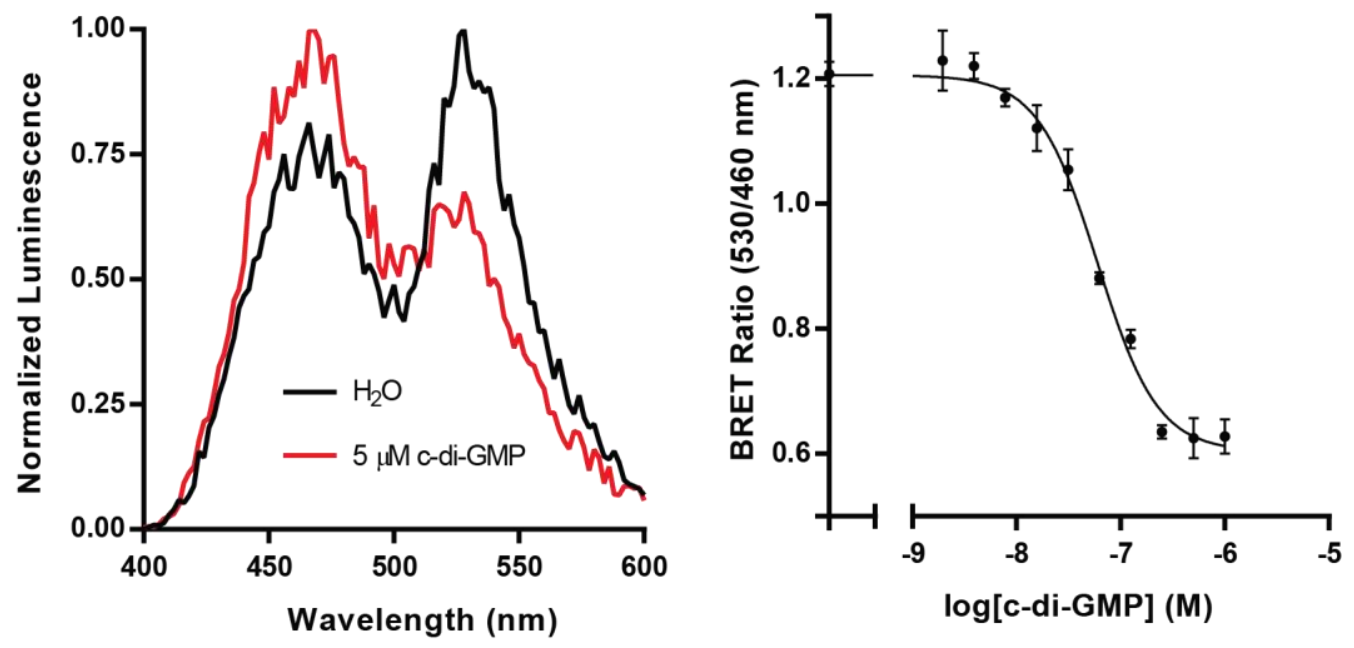

Figure 1. Design and characterization of ratiometric VYN biosensors. (a) Schematic for BRET mechanism of V-NLuc scaffold and the domain structure of the protein. Normalized luminescence emission spectra of purified V-NLuc. Data from one representative measurement. (b) Two potential mechanisms for modulation of BRET ratios by c-di-GMP binding to VYN sensors. (c) Normalized luminescence emission spectra of purified VYN-Ec in the presence and absence of c-di-GMP. Data from one representative measurement shows the biosensor follows mechanism ii. Schematic of VYN-Ec sensor shown above. (d) Binding affinity measurements for purified VYN-Ec. Data are from 3 replicates represented as mean +/- SD. 
Optimization of VYN biosensors. Four phylogenetic YcgR variants previously characterized in the YNL scaffold were selected for testing in the VYN scaffold. These YcgR variants were chosen because in the YNL scaffold they displayed high affinity, high stability, and large positive signal changes (TmYcgR, CpYcgR, and TbYcgR), or a moderate affinity and a moderate negative signal change (NtYcgR). ${ }^{15}$ Purified VYN-Tm, VYN-Nt, and VYN-Tb sensors exhibited c-di-GMP dependent changes in BRET, while the VYN-Cp sensor appeared non-responsive (Figure 2a). As expected, the functional sensors displayed higher affinities for c-di-GMP compared to VYN-Ec.

We sought to further improve the signal change of these VYN sensors through two routes: composite linker truncation and circular permutation of Venus. Both strategies are commonly used to improve signal change in the development of ratiometric sensors (FRET and BRET), but it is difficult to predict the effects on the resulting sensor. ${ }^{26,27}$ Accordingly, a small library of VYN variants was generated to screen for sensors with improved properties (Figure 2b). For linker truncation, the "composite linkers" (defined as the $\mathrm{N}$ - and C-terminal residues of Venus, YcgR, and NLuc that are not necessary for fluorescence, ligand binding, or luminescence) were analyzed. For the truncated VYN (tVYN) scaffold, 2 additional C-terminal residues from Venus and $4 \mathrm{~N}$-terminal residues from NLuc were removed. ${ }^{21}$ For YcgR $\Delta$ variants, the secondary structure prediction software SABLE was used to predict unstructured $\mathrm{N}$ - and $\mathrm{C}$-terminal residues for removal (Table S2). For circular permutations of Venus, five variants were chosen that were shown to not disrupt Venus fluorescence (Table S3). ${ }^{28}$

A library of 39 VYN variants was constructed and screened in a lysate-based assay that allows for biosensor performance to be rapidly assessed without protein purification. In this assay each individual sensor is co-expressed in $E$. coli with $\mathrm{PdeH}$, cells are lysed, cdi-GMP is added to the lysate at specified concentrations $(0,50 \mathrm{nM}$, and $5 \mu \mathrm{M})$, and BRET ratios are measured. In Figure $2 \mathrm{c}$, the $\log _{2}$ (signal fold-change) values for each sensor are reported to simplify the comparison of positive and negative signal change sensors. While no clear trends could be drawn between designs, the small set of linker truncations and circular permutations tested produced at least one BRET sensor for each YcgR protein with a signal change of $-30 \%$ or $+50 \%$ (Figure S2). Interestingly, this screen showed that seemingly small alterations in the scaffold can produce large differences in signal foldchange. The switch from tVYN-Nt to tVYN-Nt $\Delta$, for example, produced a sensor with the same relative signal fold-change, but flipped from a positive to negative change in BRET ratios (Figure 1b).

A subset of the sensors from the library was purified and characterized in vitro and was shown to span a range of affinities from $<300 \mathrm{pM}$ up to $\sim 100 \mathrm{nM}$ (Table 1). The tVYN$\mathrm{Tm} \Delta$ sensor, to our knowledge, exhibits the highest affinity cyclic dinucleotide:protein interaction ever measured, and the largest magnitude signal change ( $\Delta$ ratio of -1.04 ) out of all tested sensors in vitro. Interestingly, this sensor exhibited larger signal changes in vitro than in lysates, mostly due to truncated NLuc protein obscuring the BRET ratio in unpurified lysates (Figure S3). Given its desirable properties, we chose to apply the tVYN$\operatorname{Tm} \Delta$ sensor to develop a plate reader assay for quantification of c-di-GMP in cell extracts. 


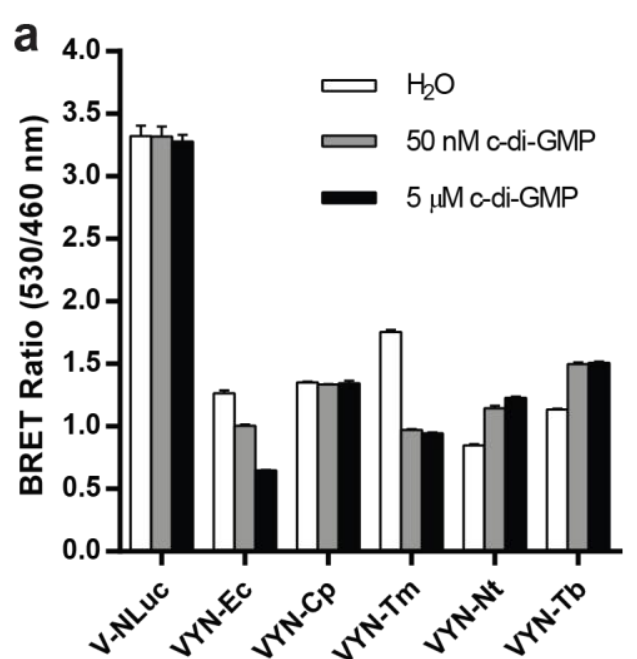

b

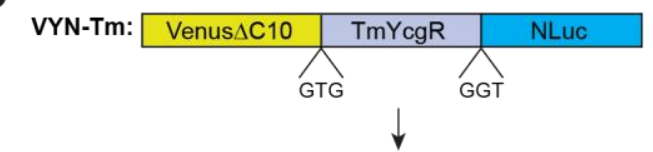

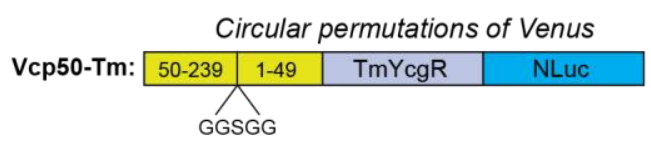

\begin{tabular}{cl|l|l|} 
& \multicolumn{2}{l}{ Truncate unstructured YcgR temini } \\
\hline VYN-Tm $\Delta:$ & Venus $\Delta$ C10 & TmYcgR $\Delta$ & NLuc \\
\hline
\end{tabular}

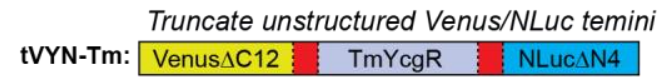

Double truncation

\begin{tabular}{l|l|l|l|}
$\operatorname{tVYN}-\mathrm{Tm} \Delta:$ & Venus $\Delta \mathrm{C12}$ & $\mathrm{TmYcgR} \Delta$ & $\mathrm{NLuc} \Delta \mathrm{N} 4$ \\
\hline
\end{tabular}

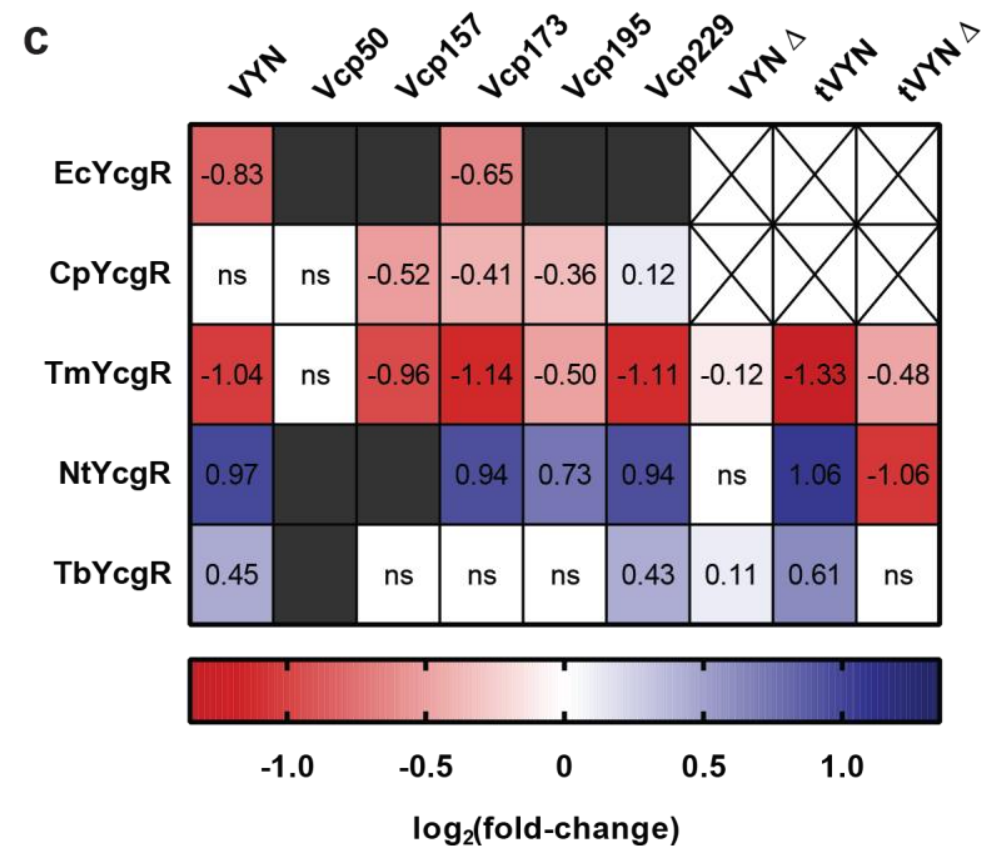

Figure 2. Optimization of VYN sensors for c-di-GMP. (a) BRET ratios of purified VYN sensors containing phylogenetic variant YcgR proteins in response to varying levels of c-di-GMP. Data are from 3 replicates represented as mean $+/$ SD. (b) Schematic representations of the domain architectures of altered VYN scaffolds, using TmYcgR as an example. Red regions highlight where truncations were made. (c) Signal fold-change (defined as BRET ratio with $5 \mu \mathrm{M}$ c-di-GMP added divided by BRET ratio with buffer added and plotted as $\log _{2}$ (fold-change)) of VYN sensor library screened in lysates. Grayed boxes = dim signal; crossed-out boxes = not tested; ns = no significant signal fold-change ( $P>0.05$ determined by Student's t-test). Data are from 4 biological replicates represented as the mean. 


\section{Table 1. Characteristics of selected VYN sensor variants}

Notes: a Data are from 3 replicates represented as mean \pm SD. bAffinity measurements were made using $300 \mathrm{pM}$ biosensor to determine $\mathrm{K}_{\mathrm{D}}$ values $<3 \mathrm{nM}$. 'Biosensor constructs were purified using an $\mathrm{N}$-terminal His6 tag, as opposed to a C-terminal His6 tag for all others.

\begin{tabular}{|c|c|c|c|c|}
\hline Sensor & $\Delta$ ratio $^{a}$ & $\%$ change $^{a}$ & $K_{D}(n M)^{a}$ & Hill coefficient ${ }^{2}$ \\
\hline tVYN-Tm $\Delta^{b, c}$ & -1.04 & $-56 \%$ & $<0.3$ & $-1.7 \pm 0.03$ \\
\hline tVYN-Tmb & -0.47 & $-50 \%$ & $0.8 \pm 0.03$ & $-1.7 \pm 0.1$ \\
\hline VYN-Tm ${ }^{b, c}$ & -0.67 & $-42 \%$ & $0.8 \pm 0.2$ & $-1.5 \pm 0.4$ \\
\hline Vcp229-Tm & -0.41 & $-44 \%$ & $2.0 \pm 0.1$ & $-1.5 \pm 0.1$ \\
\hline VYN-Tbc & 0.38 & $33 \%$ & $8.0 \pm 0.4$ & $2.4 \pm 0.3$ \\
\hline tVYN-Tb & 0.18 & $38 \%$ & $12 \pm 1$ & $2.0 \pm 0.3$ \\
\hline Vcp229-Nt & 0.22 & $52 \%$ & $14 \pm 1$ & $1.6 \pm 0.1$ \\
\hline VYN-Nt ${ }^{c}$ & 0.39 & $45 \%$ & $14 \pm 4$ & $1.4 \pm 0.4$ \\
\hline Vcp173-Nt & 0.04 & $17 \%$ & $17 \pm 4$ & $2.0 \pm 0.7$ \\
\hline tVYN-Nt & 0.24 & $56 \%$ & $20 \pm 1$ & $1.6 \pm 0.2$ \\
\hline VYN-Ec ${ }^{c}$ & -0.58 & $-48 \%$ & $50 \pm 4$ & $-1.5 \pm 0.2$ \\
\hline tVYN-Nt $\Delta$ & -1.01 & $-51 \%$ & $54 \pm 4$ & $-1.7 \pm 0.1$ \\
\hline Vcp157-Cp & -0.39 & $-33 \%$ & $96 \pm 6$ & $-1.9 \pm 0.2$ \\
\hline
\end{tabular}

Quantification of c-di-GMP in Vibrio cholerae cell extracts. The quantification of intracellular c-di-GMP levels is routinely performed using mass spectrometry (MS)based analysis of bacterial cell extracts. These methods are highly sensitive and allow for the quantitation of c-di-GMP in the picomolar or femtomolar range, depending on the detection method used. ${ }^{7-9}$ However, the sample preparation steps, long analysis time, and expertise required to perform MS-based analysis of cell extracts has limited the accessibility and throughput of these types of experiments.

Given the extremely high affinity of the tVYN-Tm $\Delta$ sensor for c-di-GMP, we predicted that it would be possible to develop a simple and robust plate reader-based assay for quantification of c-di-GMP. While the sensitivity was highest using $300 \mathrm{pM}$ biosensor (Figure 3a), quantitation of extracts was performed with $3 \mathrm{nM}$ biosensor due to improved signal intensity and stability. Under these conditions, the limit of detection (signal-to-noise ratio 3:1) of the tVYN-Tm $\Delta$ sensor was measured to be $30 \mathrm{fmol}$, which is comparable to the most sensitive established LC-MS/MS-based methods (Figure 3b), ${ }^{7,8}$ One drawback of the biosensor assay is the limited linear range ( $30 \mathrm{fmol}$ to $400 \mathrm{fmol})$, however this can be alleviated by diluting any samples that fall outside of this range (generally by 1:10$1: 20)$ or using more biosensor.

To directly compare the performance of our plate reader-based protocol to established LC-MS/MS methods, cell extract samples from $V$. cholerae were analyzed using both methods. Cell extracts were generated from three different strains of $V$. cholerae - wild- 
type (WT), wild-type lacking six DGCs $(\triangle 6 \mathrm{DGC})$, and rugose (R $\Delta \mathrm{vps} / / \mathrm{ll})$ - that were expected to produce endogenous, low, and high levels of c-di-GMP respectively. The expected differences in c-di-GMP were observed between the three strains and the quantitative data were closely correlated between the biosensor and LC-MS/MS measurements (Figure 3c; Figure S4).

We then analyzed c-di-GMP levels for $V$. cholerae grown under a variety of different conditions that have either been shown to affect or are predicted to affect endogenous cdi-GMP signaling networks within the bacterium. Altered c-di-GMP levels were observed as a result of changes in growth conditions such as $\mathrm{O}_{2}$ content, virulence factor-inducing media (AKI), pH, and salinity (Figure 3d; Figure S5). We have previously shown that cdi-GMP levels are increased at lower temperature ${ }^{29}$ and while this trend was indeed observed, the differences were not statistically significant due to high variability between biological replicates. No change was observed with iron or mucin.

Increased levels of c-di-GMP in low $\mathrm{O}_{2}$ conditions are consistent with the higher DGC activity of the diferrous form of $\mathrm{VC}_{\mathrm{c}}$ Bhr-DGC that is stable only under anaerobic conditions. ${ }^{30}$ It has been reported that $V$. cholerae cells exponentially grown in AKI did not show significantly different c-di-GMP levels compared to LB-grown cells, ${ }^{10}$ however the cells were harvested before stationary phase in that study, whereas they were harvested after stationary phase in our study. How $\mathrm{pH}$ affects c-di-GMP level in $V$. cholerae has not been shown, so further studies are required to explore this mechanism. This study also provides the first evidence that salinity directly affects c-di-GMP levels in $V$. cholerae. The non-linear results are consistent with the increased expression levels of the first gene in the c-di-GMP-regulated vps cluster, $v p s L$, at median $(0.1 \mathrm{M})$ salinity compared to low $(0 \mathrm{M})$ and high $(0.2-0.5 \mathrm{M})$ salinity. ${ }^{31}$

The limit of detection of the biosensor assay is comparable to the most sensitive established LC-MS/MS methods, but is plate-based and significantly more rapid, which makes it well-suited to high-throughput screening applications, such as monitoring enzyme activity or high throughput screening of activators/inhibitors of DGCs and PDEs. ${ }^{32}$ Accordingly, we determined the Z' factor to be $>0.5$ for the duration of 30 min after substrate addition (Figure S6), which is considered to be excellent statistical reliability for high-throughput screening. ${ }^{33}$ We even have found that the biosensor signal is sufficiently bright to be analyzed using a digital camera, which drastically reduces the cost of hardware required for c-di-GMP quantification. When applied to analysis of $V$. cholerae extracts, the biosensor assay was able to reliably quantify c-di-GMP concentrations for different strains and under different growth conditions. These results suggest that the biosensor assay will be generally applicable to the study of c-di-GMP in complex bacterial extract samples, including clinical isolates and mixed cultures. 

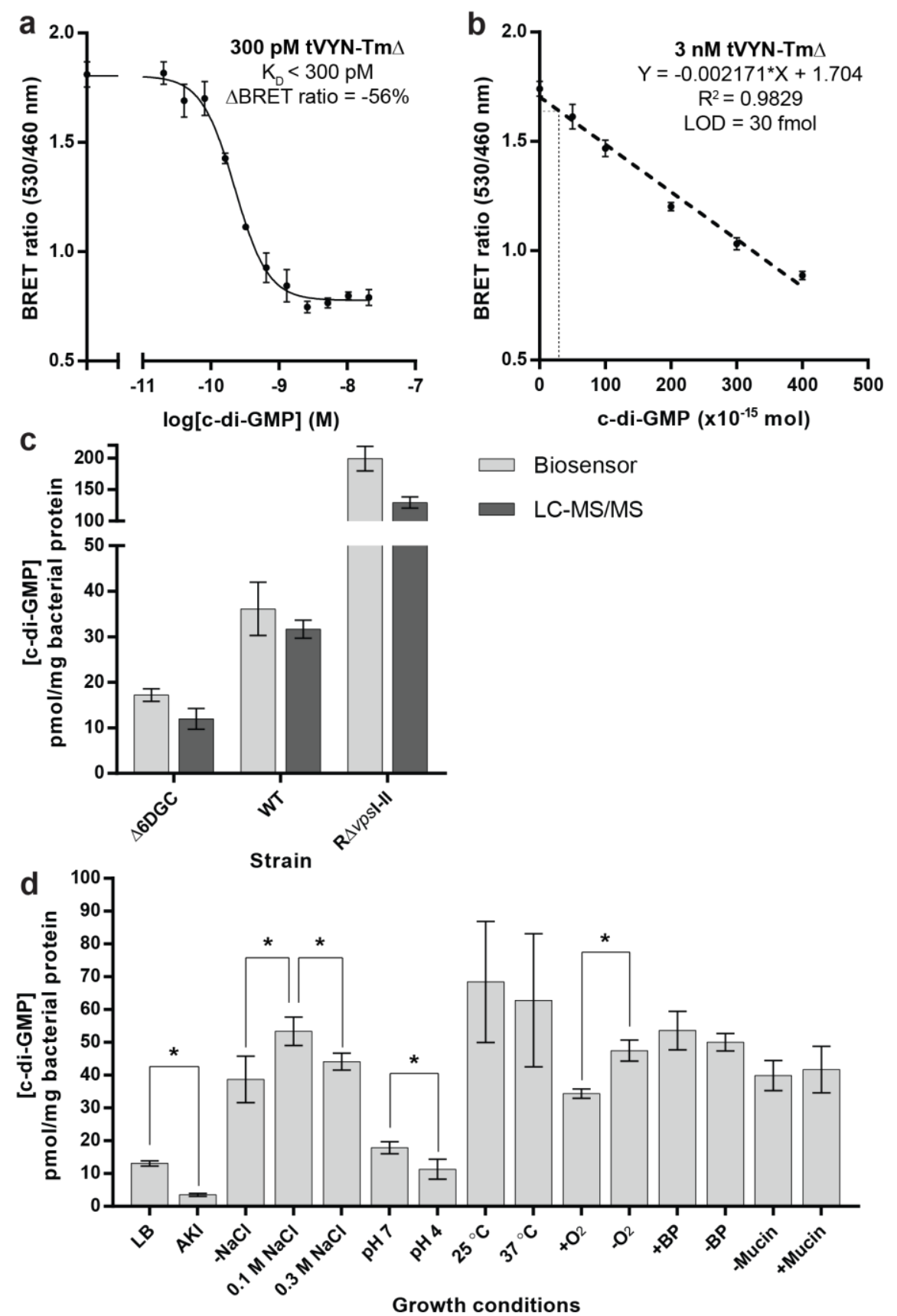

Growth conditions

Figure 3. Quantitation of c-di-GMP using tVYN-Tm $\Delta$ biosensor. (a) Binding affinity measurements for purified tVYN-Tm $\Delta$ using decreased biosensor concentration. Data are from 3 replicates represented as mean +/- SD. (b) Representative standard curve for c-di-GMP quantitation using purified tVYN-Tm $\Delta$. Data are from 6 replicates represented as mean +/- SD. (c) Quantitation of c-di-GMP in cell extracts of 3 different strains of $V$. cholerae using the tVYN$\mathrm{Tm} \Delta$ biosensor and LC-MS/MS. Data are from 3 biological replicates represented as the mean +/- SD. (d) Quantitation of c-di-GMP in cell extracts of WT V. cholerae grown under different conditions using the tVYN-Tm $\Delta$ biosensor. Asterisks $\left(^{*}\right)$ denote significant changes in c-di-GMP between growth conditions ( $P<0.05$ determined by Student's t-test). Data are from 3 biological replicates represented as the mean $+/-$ SD, except for 25 and $37^{\circ} \mathrm{C}$ conditions, which are from 6 biological replicates. 
Live-cell measurements of c-di-GMP using IVIS imager and tissue-like phantom model. With our prior YNL-based sensors we encountered difficulties in making live cell measurements, likely due to changes in luminescent substrate availability and biosensor expression between different conditions that complicated normalization of the intensity-based signal (Figure S7). ${ }^{15}$ To test if the ratiometric VYN biosensors alleviate these issues for live cell measurements, a subset of sensors were co-expressed in BL21 Star (DE3) E. coli with a PDE (PdeH - low c-di-GMP), an inactive diguanylate cyclase (DGC) as a control (WspR-G249A - endogenous c-di-GMP), or a constitutively active DGC (WspR-D70E - elevated c-di-GMP). Encouragingly, many of the sensors showed significant changes in BRET ratio between low or endogenous c-di-GMP conditions versus elevated c-di-GMP conditions (Figure 4).

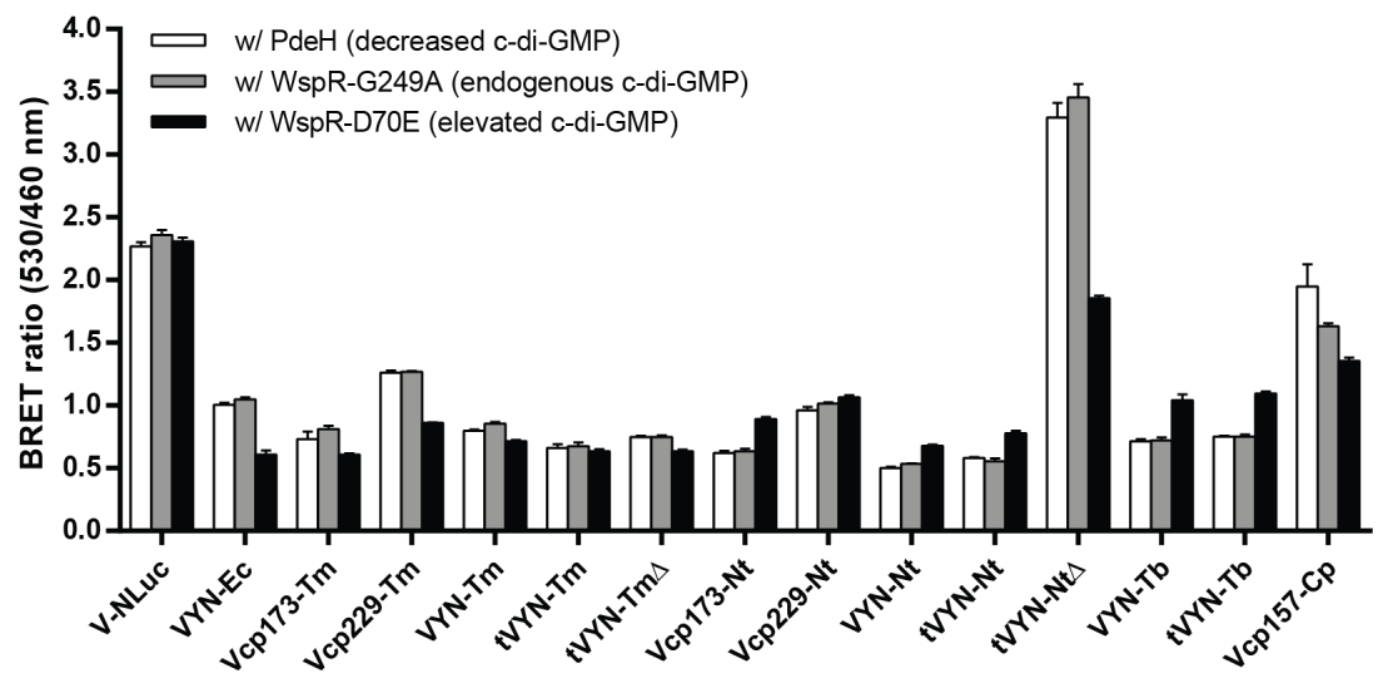

Figure 4. Live cell measurements of c-di-GMP using VYN sensors. BRET ratios of $E$. coli cells co-expressing VYN biosensors with PdeH, WspR-G249A, or WspR-D70E. Data are from 4 biological replicates represented as mean $+/$ - SD.

One of our long-term goals is to monitor signaling activity of bacterial cells in real time during host infection. An initial proof-of-concept experiment was to validate the signal intensity and BRET signal changes of our sensors measured in an instrument routinely used for non-invasive small animal imaging, a Xenogen IVIS 100, with conventional filter sets and settings. Selected sensors were co-expressed in BL21 Star (DE3) E. coli cells with WspR-G249A or WspR-D70E to produce endogenous or elevated c-di-GMP levels, respectively. Cells were prepared in the same manner as for plate reader experiments, then images were captured sequentially after luminescent substrate addition using no emission filter and standard 500 and $540 \mathrm{~nm}$ emission filters on the IVIS. The total flux (photons/sec) from each well in the $540 \mathrm{~nm}$ and $500 \mathrm{~nm}$ filter images was used to calculate BRET ratios (Figure 5a).

The raw BRET ratio values are different between IVIS and plate reader instruments, likely due to less optimal emission filters for the biosensor on the IVIS. Nevertheless, the 
changes in BRET ratio in response to c-di-GMP were faithfully reproduced for VYN-Ec and tVYN-Nt $\Delta$ sensors with 2- and 200-fold fold dilution of cells (Figures 5a, S8a). With much higher dilution (20,000-fold) and thus lower signal-to-noise, the tVYN-Nt $\Delta$ sensor but not VYN-Ec maintained the expected response to c-di-GMP, due to its larger magnitude signal change ( $\triangle \mathrm{BRET} \sim 1$ vs. $\sim 0.5)$.

To further extend the proof-of-concept, tissue-like phantom materials were utilized to mimic the light absorption and scattering of living tissue. ${ }^{34}$ These types of tissue phantoms have recently been used as a benchmark to compare photon output of luminescent protein systems within deep tissues. ${ }^{35}$ The 96 -well plate containing bacterial cells was covered with $1.5 \mathrm{~mm}$ thick tissue phantom prior to image capture (Figure S9). While luminescent intensity and BRET ratios were generally lower with application of the tissue phantom (the latter due to hemoglobin absorbing more strongly at $540 \mathrm{~nm}$ than $500 \mathrm{~nm}$ ), ${ }^{36}$ the overall results were similar to without phantom (Figure $5 \mathrm{~b}$ ). Luminescent signal still could be detected down to 20,000-fold dilution for all samples, and the tVYN-Nt $\Delta$ sensor displayed significant response to c-di-GMP down to that dilution (Figure 5c).

To determine the amount of bacteria monitored in the IVIS experiments, the number of colony-forming units (CFUs) was measured for representative cultures co-expressing the tVYN-Nt $\Delta$ biosensor and WspR-G249A or WspR-D70E. Cells were prepared as before and then spotted onto LB/Agar plates containing no antibiotic, carbenicillin (Carb), kanamycin (Kan), or both Carb and Kan, the last condition being the overnight growth conditions used for all live-cell experiments. Results from plates with no antibiotics show that there are $\sim 10^{8}$ E. coli in each well for 2 -fold diluted cultures, and $\sim 10^{6}$ and $\sim 10^{4}$ cells in the 200-fold and 20,000-fold diluted cultures, respectively, as expected. However, comparisons to antibiotic plates reveal that $\sim 90 \%$ of these cells have lost both biosensor and WspR expression plasmids after overnight growth (Figures S10). Thus, the actual number of bacteria producing luminescent signal is only $10 \%$ of the total. Given the tVYN$\mathrm{Nt} \Delta$ sensor is capable of imaging c-di-GMP levels in 20,000-fold diluted cultures in a tissue-like model, this corresponds to as few as $\sim 10^{3}$ biosensor-expressing bacterial cells. In comparison, a $V$. cholerae infection model of infant mice presented $10^{4}$ to $10^{5}$ bacteria in the small intestine after infection. ${ }^{37}$

Importantly, we found that the BRET ratio signal for tVYN-Nt $\Delta$ sensor under the tissue phantom remained stable over the course of an hour after luminescent substrate addition, even while overall signal intensity decayed as substrate was consumed (Figure S8c). While the tissue phantom model does not account for substrate distribution in vivo, NLuc and furimazine previously have been applied to study the spread of pathogens in mice in real time..$^{38,39}$ Our experiments were performed using coelenterazine-h as the luminescent substrate, so even brighter luminescent signal in vivo should be possible with furimazine, which produces higher luminescent output than coelenterazine- $h .{ }^{17}$ Beyond the hour timescale, longer term experiments (days or weeks) could be carried out with repeated administration of luminescent substrate. 

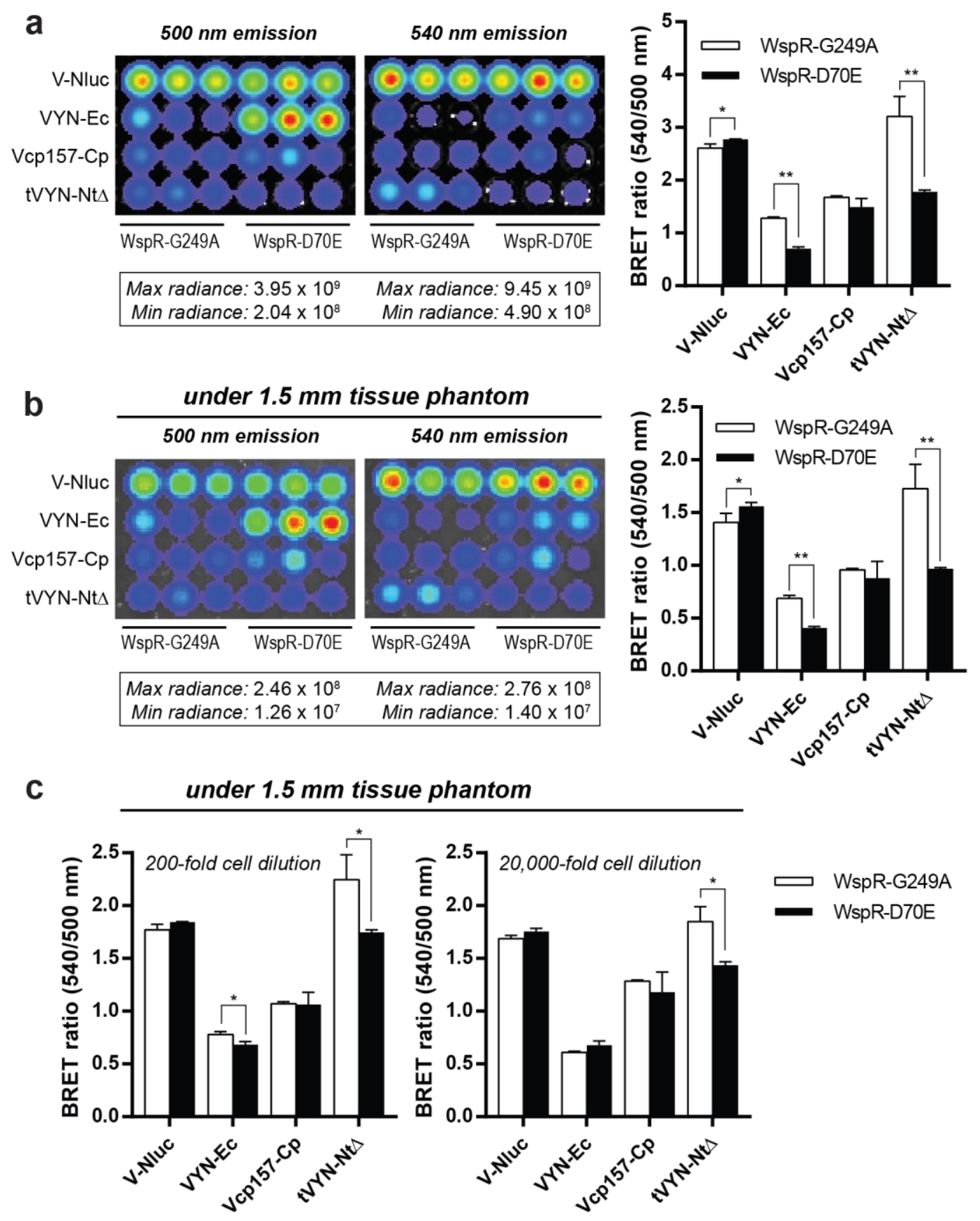

Figure 5. Live cell measurements of c-di-GMP using a tissue phantom model. (a) Luminescent images of 2-fold diluted $E$. coli cultures co-expressing VYN biosensors with WspRG249A or WspR-D70E captured by an IVIS 100, and the BRET values calculated from the total radiance in each well. Maximum and minimum radiance values (photons $/ \mathrm{sec} / \mathrm{cm}^{2} / \mathrm{steradian}$ ) captured for each image are shown. (b) Same as part (a), except plate was covered with a 1.5 $\mathrm{mm}$ thick tissue phantom prior to image capture. (c) BRET ratios of serially diluted $E$. coli cultures co-expressing VYN biosensors with WspR-G249A or WspR-D70E calculated from the radiance of each well. Plate was covered with a $1.5 \mathrm{~mm}$ thick tissue phantom prior to image capture. For all graphs, data are from 3 biological replicates represented as mean +/- SD. Asterisks $\left({ }^{*}\right)$ denote significant changes in BRET ratio ( ${ }^{*} \mathrm{P}<0.05,{ }^{* *} \mathrm{P}<0.005$ determined by Student's t-test). 


\section{CONCLUSIONS}

The work here presents, to our knowledge, the first ratiometric, luminescent biosensors developed to study bacterial signaling. The highest affinity VYN biosensor, tVYN-Tm $\Delta$, can serve as an easy-to-use diagnostic reagent for quantifying c-di-GMP levels from bacterial extracts, with comparable sensitivity to LC-MS/MS. Furthermore, as a genetically encodable tool, the tVYN-Nt $\Delta$ sensor holds considerable promise for monitoring c-di-GMP dynamics in real-time during host infection using a standard small animal imaging system. More broadly, this study demonstrates how to develop and characterize luminescent biosensors that are directed towards studying bacterial activity in complex environments. 


\section{MATERIALS AND METHODS}

Chemiluminescence measurements with purified protein. Briefly, proteins and ligands were prepared in opaque white 96-well LUMITRAC 600 plates (Grenier) in assay buffer [50 mM HEPES (pH 7.2), $100 \mathrm{mM} \mathrm{KCl,} 10 \mathrm{mM}$ DTT, 0.1\% BSA]. Unless otherwise noted, all measurements using purified protein were made using $3 \mathrm{nM}$ sensor in $100 \mu \mathrm{L}$ total reaction volume, then incubated at $28 \stackrel{\circ}{\circ} \mathrm{C}$ for at least 10 min to reach binding equilibrium. Chemiluminescent substrate was prepared by diluting coelenterazine-h to 60 $\mu \mathrm{M}$ in reagent buffer [50 mM HEPES ( $\mathrm{pH} 7.2), 100 \mathrm{mM} \mathrm{KCl}, 300 \mathrm{mM}$ ascorbate], and equilibrating the solution at RT for at least 30 min. Unless otherwise noted, all biosensor measurements were taken at $28{ }^{\circ} \mathrm{C}$ in a SpectraMax i3x plate reader (Molecular Devices) after manually adding $20 \mu \mathrm{L}$ of chemiluminescent substrate. Emission intensities were measured at 460 and $530 \mathrm{~nm}$ with $200 \mathrm{~ms}$ integration time at $30 \mathrm{~s}$ intervals for $10 \mathrm{~min}$ after chemiluminescent substrate addition. In general, BRET ratios were calculated using emission values obtained $2 \mathrm{~min}$ after substrate addition. For emission spectrum measurements, emission intensities were measured over the range of 400-600 nm in steps of $2 \mathrm{~nm}$.

Lysate-based assay for biosensor activity. The lysate-based assay was carried out as previously described, with minor modifications. ${ }^{15}$ Single colonies of BL21 Star (DE3) E. coli cells co-transformed with pET21-biosensor and pCOLA-PdeH plasmids were resuspended in $500 \mu \mathrm{L}$ of $\mathrm{P}-0.5 \mathrm{G}$ non-inducing media $[0.5 \%$ glucose, $25 \mathrm{mM}$ $\left.\left(\mathrm{NH}_{4}\right)_{2} \mathrm{SO}_{4}, 50 \mathrm{mM} \mathrm{KH}_{2} \mathrm{PO}_{4}, 50 \mathrm{mM} \mathrm{Na} \mathrm{HPO}_{4}, 1 \mathrm{mM} \mathrm{MgSO}\right]^{40}$ supplemented with 50 $\mu \mathrm{g} / \mathrm{mL}$ carbenicillin and $100 \mu \mathrm{g} / \mathrm{mL}$ kanamycin in $2.2 \mathrm{~mL}$ 96-well deep-well plates (VWR). Precultures were grown at $37^{\circ} \mathrm{C}, 340 \mathrm{rpm}$ for $24 \mathrm{~h}$ at which point $5 \mu \mathrm{L}$ of each was used to inoculate $500 \mu \mathrm{L}$ of ZYP-5052 autoinduction media [25 mM (NH $)_{2} \mathrm{SO}_{4}, 50 \mathrm{mM} \mathrm{KH}_{2} \mathrm{PO}_{4}$, $50 \mathrm{mM} \mathrm{Na}_{2} \mathrm{HPO}_{4}, 1 \mathrm{mM} \mathrm{MgSO}_{4}, 0.5 \%$ (v/v) glycerol, $0.05 \%$ glucose, $0.2 \%$ a-lactose, $1 \%$ tryptone, and $0.5 \%$ yeast extract] ${ }^{40}$ supplemented with $50 \mu \mathrm{g} / \mathrm{mL}$ carbenicillin and 100 $\mu \mathrm{g} / \mathrm{mL}$ kanamycin. Cultures were grown in ZYP-5052 autoinduction media at $37^{\circ} \mathrm{C}, 340$ $\mathrm{rpm}$ for $20 \mathrm{~h}$ to express the biosensors, then harvested by centrifugation at $4700 \mathrm{rpm}$ for 10 minutes at $4 \stackrel{\circ}{\circ}$. Lysates were prepared by removing the supernatant media and resuspending cell pellets in $500 \mu \mathrm{L}$ of screening buffer [50 mM Tris $(\mathrm{pH} 7.5), 100 \mathrm{mM} \mathrm{KCl}$, $5 \%$ glycerol, $2 \mathrm{mM}$ EDTA, $300 \mu \mathrm{g} / \mathrm{mL}$ lysozyme, $1 \mathrm{mM} \mathrm{PMSF].} \mathrm{Cells} \mathrm{were} \mathrm{incubated} \mathrm{for}$ $1 \mathrm{~h}$ at $4{ }^{\circ} \mathrm{C}$ to gently lyse, and total lysates were centrifuged for $40 \mathrm{~min}$ at $4700 \mathrm{rpm}$ at 4 ${ }^{\circ} \mathrm{C}$ to generate clarified lysates.

For chemiluminescence measurements, $5 \mu \mathrm{L}$ of clarified lysate was mixed with 85 $\mu \mathrm{L}$ screening buffer (-lysozyme, -PMSF) and $10 \mu \mathrm{L}$ of either buffer, $500 \mathrm{nM}$ c-di-GMP, or $50 \mu \mathrm{M}$ c-di-GMP [in screening buffer (-lysozyme, -PMSF)] in opaque white 96-well LUMITRAC 600 plates (Greiner) to generate final concentrations of $0,50 \mathrm{nM}$, or $5 \mu \mathrm{M}$ cdi-GMP. Chemiluminescence was measured using the same method described for purified protein, except BRET ratios were calculated using emission values obtained 1 min after substrate addition. 
Vibrio cholerae strains and growth conditions. Vibrio cholerae O1 El Tor A1552 was used as the wild-type strain and two $V$. cholerae strains, $\triangle 6 \mathrm{DGC}^{29}$ and $\mathrm{R} \Delta$ vpsl-II, ${ }^{41}$ were used as reference strains with low and high cellular c-di-GMP level, respectively. Strains were grown in Luria-Bertani (LB) medium [1\% tryptone, $0.5 \%$ yeast extract, 0.2 $\mathrm{M} \mathrm{NaCl} ; \mathrm{pH} 7.5]$ with constant shaking at $200 \mathrm{rpm}$ at $37^{\circ} \mathrm{C}$ unless otherwise indicated. To test the effects of salt concentration, LB supplemented with different concentrations of $\mathrm{NaCl}(0,0.1$, and $0.3 \mathrm{M})$ were used..$^{42}$ To test the effects of different growth temperature ${ }^{29}$ and oxygen availability, the diluted cultures were grown at 25 and $37^{\circ} \mathrm{C}$ to $\mathrm{OD}_{600} \sim 0.5$ or aerobically and anaerobically (in a Vinyl Anaerobic Airlock Chamber, Coy Laboratory Products) to $\mathrm{OD}_{600} \sim 0.3$. To test the effects of mucin addition ${ }^{43}$ and iron depletion, ${ }^{44}$ overnight-grown cultures were inoculated in a 1:200 dilution in LB supplemented with different components [0.4\% (w/v) of bovine submaxillary gland mucin (Sigma-Aldrich), or $200 \mu \mathrm{M}$ of 2,2'-dipyridyl (Alfa Aesar), respectively] and grown to OD600 $\sim 0.5$. To test virulence-inducing conditions, overnight-grown cultures were inoculated in a 1:200 dilution in LB and in a 1:100 dilution in AKI [1.5\% Bacto peptone, $0.4 \%$ yeast extract, $0.5 \% \mathrm{NaCl}, 0.3 \% \mathrm{NaHCO}_{3}$ ]. LB cultures were grown overnight with shaking at $220 \mathrm{rpm}$ at $37^{\circ} \mathrm{C}$. AKI cultures were grown statically at $37^{\circ} \mathrm{C}$ for 4 hours followed by shaking at 220 rpm at $37^{\circ} \mathrm{C}$ overnight. ${ }^{45}$ To test the effect of acidic conditions, overnight-grown cultures were inoculated in a 1:200 dilution in LB ( $\mathrm{pH} 7$ ), grown to OD600 $\sim 0.5$, and centrifuged at $1500 \times \mathrm{g}$ for 7 minutes. Cell pellets were adapted by resuspending in $\mathrm{LB}(\mathrm{pH} 5.7)$ and incubating for 1 hour. Adapted cells were centrifugated and resuspended in LB $(\mathrm{pH} 4)$ followed by 1 hour incubation. ${ }^{46}$

Live cell measurements with biosensor co-expression. Single colonies of BL21 Star (DE3) E. coli cells co-transformed with pET21-biosensor and pCOLA-PdeH, pCOLA-WspR-G249A, or pCOLA-WspR-D70E plasmids were resuspended and grown in the same manner as previously described for the lysate-based assay. After growth and induction of expression, cells were centrifuged, supernatant media was removed, and cell pellets were resuspended in $500 \mu \mathrm{L}$ PBS [137 mM NaCl, $2.7 \mathrm{mM} \mathrm{KCl}, 10 \mathrm{mM} \mathrm{Na} 2 \mathrm{HPO}_{4}$, $1.8 \mathrm{mM} \mathrm{KH}_{2} \mathrm{PO}_{4}(\mathrm{pH} 7.4)$ ]. For chemiluminescence measurements, cells were diluted 2fold with PBS in an opaque 96-well LUMITRAC 600 plate (Greiner) to a final volume of $100 \mu \mathrm{L}$. Chemiluminescent substrate was added and emission intensities were measured in the same way as described for purified protein. BRET ratios were calculated using emission values obtained 5 min after substrate addition.

Live cell measurements in tissue-like phantom model. Tissue-like phantoms were prepared as described previously. ${ }^{34}$ Briefly, the phantom solution mixture was prepared with $10 \%$ gelatin, $170 \mu \mathrm{M}$ bovine hemoglobin, and $1 \%$ intralipid in TBS-azide buffer [50 mM Tris- $\mathrm{HCl}(\mathrm{pH} 7.4), 150 \mathrm{mM} \mathrm{NaCl}, 0.1 \% \mathrm{NaN}_{3}$ ]. Phantoms were poured to the desired thickness of $1.5 \mathrm{~mm}$ between glass plates to ensure uniformity, then stored at $4{ }^{\circ} \mathrm{C}$.

Chemiluminescence measurements were carried out in a Xenogen IVIS 100 Bioluminescent Imager at the Huntsman Cancer Institute Center for Quantitative Cancer 
Imaging. Cells were grown and prepared in the same way as the live cell co-expression experiments but were diluted 2-fold, 200-fold, and 20,000-fold in PBS in opaque black 96well assay plates (CoStar). To image the plates, $20 \mu \mathrm{L}$ of chemiluminescent substrate was added to each well and plates were placed in the chamber. Luminescent images were captured sequentially using no filter, a $500 \mathrm{~nm}$ filter, and a $540 \mathrm{~nm}$ filter within a 13 $\mathrm{cm}$ field of view. The instrument was set to auto-adjust settings to ensure maximum signal for each image (exposure time of $0.5-60 \mathrm{~s}$, binning of $1 \mathrm{x}-16 \mathrm{x}, \mathrm{f} / \mathrm{stop}$ of 1 ). For experiments with tissue-like phantom model, the wells were covered with a phantom immediately after addition of chemiluminescent substrate, and luminescent images were captured as before. For image analysis, a 12x8 ROI grid was applied to each image and used to calculate the flux (photons/s) for each individual well. For time course images, the same plate was repeatedly imaged for up to an hour after the initial addition of chemiluminescent substrate.

\section{FUNDING}

This work was supported in part by NIH grant R01 GM124589 (to M.C.H.), NIH grant R01 Al102584 (to F.H.Y.), NIH training grant T32 GM066698 (for A.B.D.), and the Community Science Program project 1473 (to M.C.H.) by the Joint Genome Institute, a DOE Office of Science User Facility, that is supported by the Office of Science of the U.S. Department of Energy under Contract No. DE-AC02-05CH11231. 


\section{REFERENCES}

(1) Ross, P., Weinhouse, H., Aloni, Y., Michaeli, D., Weinberger-Ohana, P., Mayer, R., Braun, S., de Vroom, E., van der Marel, G. A., van Boom, J. H., and Benziman, M. (1987) Regulation of cellulose synthesis in Acetobacter xylinum by cyclic diguanylic acid. Nature 325, 279-281.

(2) Romling, U., Galperin, M. Y., and Gomelsky, M. (2013) Cyclic di-GMP: the first 25 years of a universal bacterial second messenger. Microbiol. Mol. Biol. Rev. 77, 1-52.

(3) Jenal, U., Reinders, A., and Lori, C. (2017) Cyclic di-GMP: Second messenger extraordinaire. Nat. Rev. Microbiol. 15, 271-284.

(4) Hall, C. L., and Lee, V. T. (2018) Cyclic-di-GMP regulation of virulence in bacterial pathogens. Wiley Interdiscip. Rev. RNA 9, 1-19.

(5) Wolfe, A. J., and Berg, H. C. (1989) Migration of bacteria in semisolid agar. Proc. Natl. Acad. Sci. 86, 6973-6977.

(6) O'Toole, G., Pratt, L., Watnick, P., Newman, D., Weaver, V., and Kolter, R. (1999) Genetic Approaches to Study of Biofilms. Methods Enzymol. 310, 91-109.

(7) Petrova, O. E., and Sauer, K. (2017) High-Performance Liquid Chromatography (HPLC)-Based Detection and Quantitation of Cellular c-di-GMP, in Methods in Molecular Biology, pp 33-43.

(8) Burhenne, H., and Kaever, V. (2013) Quantification of Cyclic Dinucleotides by Reversed-Phase LC-MS/MS, in Methods in Molecular Biology, pp 27-37.

(9) Spangler, C., Böhm, A., Jenal, U., Seifert, R., and Kaever, V. (2010) A liquid chromatography-coupled tandem mass spectrometry method for quantitation of cyclic di-guanosine monophosphate. J. Microbiol. Methods 81, 226-231.

(10) Koestler, B. J., and Waters, C. M. (2013) Exploring environmental control of cyclic di-gmp signaling in vibrio cholerae by using the ex vivo lysate cyclic di-GMP assay(TELCA). Appl. Environ. Microbiol. 79, 5233-5241.

(11) Christen, M., Kulasekara, H. D., Christen, B., Kulasekara, B. R., Hoffman, L. R., and Miller, S. I. (2010) Asymmetrical distribution of the second messenger c-di-GMP upon bacterial cell division. Science 328, 1295-1297.

(12) Ho, C. L., Chong, K. S. J., Oppong, J. A., Chuah, M. L. C., Tan, S. M., and Liang, Z. X. (2013) Visualizing the perturbation of cellular cyclic di-GMP levels in bacterial cells. J. Am. Chem. Soc. 135, 566-569.

(13) Wang, X. C., Wilson, S. C., and Hammond, M. C. (2016) Next-generation fluorescent RNA biosensors enable anaerobic detection of cyclic di-GMP. Nucleic Acids Res. 44, e139.

(14) Yeo, J., Dippel, A. B., Wang, X. C., and Hammond, M. C. (2017) In Vivo Biochemistry: Single-cell dynamics of cyclic di-GMP in E. coli in response to zinc overload. Biochemistry 57, 108-116. 
(15) Dippel, A. B., Anderson, W. A., Evans, R. S., Deutsch, S., and Hammond, M. C. (2018) Chemiluminescent Biosensors for Detection of Second Messenger Cyclic diGMP. ACS Chem. Biol. 13, 1872-1879.

(16) Saito, K., Chang, Y.-F., Horikawa, K., Hatsugai, N., Higuchi, Y., Hashida, M., Yoshida, Y., Matsuda, T., Arai, Y., and Nagai, T. (2012) Luminescent proteins for highspeed single-cell and whole-body imaging. Nat. Commun. 3, 1262.

(17) Hall, M. P., Unch, J., Binkowski, B. F., Valley, M. P., Butler, B. L., Wood, M. G., Otto, P., Zimmerman, K., Vidugiris, G., MacHleidt, T., Robers, M. B., Benink, H. a., Eggers, C. T., Slater, M. R., Meisenheimer, P. L., Klaubert, D. H., Fan, F., Encell, L. P., and Wood, K. V. (2012) Engineered luciferase reporter from a deep sea shrimp utilizing a novel imidazopyrazinone substrate. ACS Chem. Biol. 7, 1848-1857.

(18) Yang, J., Cumberbatch, D., Centanni, S., Shi, S., Winder, D., Webb, D., and Johnson, C. H. (2016) Coupling optogenetic stimulation with NanoLuc-based luminescence (BRET) Ca++ sensing. Nat. Commun. 7, 13268.

(19) Aper, S. J. A., Dierickx, P., and Merkx, M. (2016) Dual readout BRET/FRETsensors for measuring intracellular zinc. ACS Chem. Biol. 11, 2854-2864.

(20) Inagaki, S., Tsutsui, H., Suzuki, K., Agetsuma, M., Arai, Y., Jinno, Y., Bai, G., Daniels, M. J., Okamura, Y., Matsuda, T., and Nagai, T. (2017) Genetically encoded bioluminescent voltage indicator for multi-purpose use in wide range of bioimaging. Sci. Rep. 7, 1-11.

(21) Suzuki, K., Kimura, T., Shinoda, H., Bai, G., Daniels, M. J., Arai, Y., Nakano, M., and Nagai, T. (2016) Five colour variants of bright luminescent protein for real-time multicolour bioimaging. Nat. Commun. 7, 13718.

(22) Ryjenkov, D. A., Simm, R., Römling, U., and Gomelsky, M. (2006) The PilZ domain is a receptor for the second messenger c-di-GMP: The PilZ domain protein YcgR controls motility in enterobacteria. J. Biol. Chem. 281, 30310-30314.

(23) Ko, J., Ryu, K. S., Kim, H., Shin, J. S., Lee, J. O., Cheong, C., and Choi, B. S. (2010) Structure of PP4397 Reveals the Molecular Basis for Different c-di-GMP Binding Modes by Pilz Domain Proteins. J. Mol. Biol. 398, 97-110.

(24) Benach, J., Swaminathan, S. S., Tamayo, R., Handelman, S. K., Folta-Stogniew, E., Ramos, J. E., Forouhar, F., Neely, H., Seetharaman, J., Camilli, A., and Hunt, J. F. (2007) The structural basis of cyclic diguanylate signal transduction by PilZ domains. EMBO J. 26, 5153-66.

(25) Pultz, I. S., Christen, M., Kulasekara, H. D., Kennard, A., Kulasekara, B., and Miller, S. I. (2012) The response threshold of Salmonella PilZ domain proteins is determined by their binding affinities for c-di-GMP. Mol. Microbiol. 86, 1424-1440.

(26) Deuschle, K., Okumoto, S., Fehr, M., Looger, L. L., Kozhukh, L., and Frommer, W. B. (2005) Construction and optimization of a family of genetically encoded metabolite sensors by semirational protein engineering. Protein Sci. 14, 2304-2314.

(27) van Rosmalen, M., Krom, M., and Merkx, M. (2017) Tuning the Flexibility of 
Glycine-Serine Linkers To Allow Rational Design of Multidomain Proteins. Biochemistry $56,6565-6574$.

(28) Nagai, T., Yamada, S., Tominaga, T., Ichikawa, M., and Miyawaki, A. (2004) Expanded dynamic range of fluorescent indicators for $\mathrm{Ca} 2+$ by circularly permuted yellow fluorescent proteins. Proc. Natl. Acad. Sci. 101, 10554-10559.

(29) Townsley, L., and Yildiz, F. H. (2015) Temperature affects c-di-GMP signalling and biofilm formation in Vibrio cholerae. Environ. Microbiol. 17, 4290-4305.

(30) Schaller, R. A., Ali, S. K., Klose, K. E., and Kurtz, D. M. (2012) A bacterial hemerythrin domain regulates the activity of a Vibrio cholerae diguanylate cyclase. Biochemistry 51, 8563-8570.

(31) Shikuma, N. J., and Yildiz, F. H. (2009) Identification and characterization of OscR, a transcriptional regulator involved in osmolarity adaptation in Vibrio cholerae. J. Bacteriol. 191, 4082-4096.

(32) Opoku-Temeng, C., Zhou, J., Zheng, Y., Su, J., and Sintim, H. O. (2016) Cyclic dinucleotide (c-di-GMP, c-di-AMP, and cGAMP) signalings have come of age to be inhibited by small molecules. Chem. Commun. 52, 9327-9342.

(33) Ji-Hu, Z., Thomas, D. Y. C., and Kevin, R. O. (1999) A Simple Statistical Parameter for Use in Evaluation and Validation of High Throughput Screening Assays. J. Biomol. Screen. 4, 67-73.

(34) De Grand, A. M., Lomnes, S. J., Lee, D. S., Pietrzykowski, M., Ohnishi, S., Morgan, T. G., Gogbashian, A., Laurence, R. G., and Frangioni, J. V. (2006) Tissue-like phantoms for near-infrared fluorescence imaging system assessment and the training of surgeons. J. Biomed. Opt. 11, 014007.

(35) Chu, J., Oh, Y., Sens, A., Ataie, N., Dana, H., Macklin, J. J., Laviv, T., Welf, E. S., Dean, K. M., Zhang, F., Kim, B. B., Tang, C. T., Hu, M., Baird, M. A., Davidson, M. W., Kay, M. A., Fiolka, R., Yasuda, R., Kim, D. S., Ng, H.-L., and Lin, M. Z. (2016) A bright cyan-excitable orange fluorescent protein facilitates dual-emission microscopy and enhances bioluminescence imaging in vivo. Nat. Biotechnol. 34, 760-767.

(36) Zhao, H., Doyle, T. C., Coquoz, O., Kalish, F., Rice, B. W., and Contag, C. H. (2005) Emission spectra of bioluminescent reporters and interaction with mammalian tissue determine the sensitivity of detection in vivo. J. Biomed. Opt. 10, 041210.

(37) Tamayo, R., Patimalla, B., and Camilli, A. (2010) Growth in a biofilm induces a hyperinfectious phenotype in Vibrio cholerae. Infect. Immun. 78, 3560-3569.

(38) Tran, V., Moser, L. A., Poole, D. S., and Mehle, A. (2013) Highly Sensitive RealTime In Vivo Imaging of an Influenza Reporter Virus Reveals Dynamics of Replication and Spread. J. Virol. 87, 13321-13329.

(39) Silberstein, E., Serna, C., Fragoso, S. P., Nagarkatti, R., and Debrabant, A. (2018) A novel nanoluciferase-based system to monitor Trypanosoma cruzi infection in mice by bioluminescence imaging. PLoS One 13,1-21. 
(40) Studier, F. W. (2005) Protein production by auto-induction in high-density shaking cultures. Protein Expr. Purif. 41, 207-234.

(41) Fong, J. C. N., Syed, K. A., Klose, K. E., and Yildiz, F. H. (2010) Role of Vibrio polysaccharide (vps) genes in VPS production, biofilm formation and Vibrio cholerae pathogenesis. Microbiology 156, 2757-2769.

(42) Shikuma, N. J., Davis, K. R., Fong, J. N. C., and Yildiz, F. H. (2013) The transcriptional regulator, CosR, controls compatible solute biosynthesis and transport, motility and biofilm formation in Vibrio cholerae. Environ. Microbiol. 15, 1387-1399.

(43) Silva, A. J., Pham, K., and Benitez, J. A. (2003) Haemagglutinin/protease expression and mucin gel penetration in El Tor biotype Vibrio cholerae. Microbiology 149, 1883-1891.

(44) Rogers, M. B., Sexton, J. A., DeCastro, G. J., and Calderwood, S. B. (2000) Identification of an operon required for ferrichrome iron utilization in Vibrio cholerae. J. Bacteriol. 182, 2350-2353.

(45) Iwanaga, M., Yamamoto, K., Higa, N., Ichinose, Y., Nakasone, N., and Tanabe, M. (1986) Culture Conditions for Stimulating Cholera Toxin Production by Vibrio cholerae 01 El Tor. Microbiol. Immunol. 30, 1075-1083.

(46) Merrell, D. S., Hava, D. L., and Camilli, A. (2002) Identification of novel factors involved in colonization and acid tolerance of Vibrio cholerae. Mol. Microbiol. 43, 14711491. 